\title{
INFLUÊNCIA DOS NÍVEIS DE PROLACTINA E TAMANHO TUMORAL NA FUNÇÃO HIPOFISÁRIA PÓS-OPERATÓRIA EM MACROADENOMAS HIPOFISÁRIOS CLINICAMENTE NÃO-FUNCIONANTES
}

\author{
Ana Luiza Vidal Fonseca', Leila Chimelli², Mario José C. Felippe Santos ${ }^{3}$, \\ Alair Augusto S.M. Damas dos Santos', Alice Helena Dutra Violante ${ }^{5}$
}

\begin{abstract}
RESUMO - Objetivo: Estudar a influência da hiperprolactinemia e de tamanho tumoral na função hipofisária em macroadenomas hipofisários clinicamente não funcionantes. Métodos: Foram analisados 23 pacientes com macroadenomas hipofisários clinicamente não funcionantes, com exames de imagem (tomografia computadorizada ou ressonância magnética) e dosagens hormonais basais; 16 tinham provas de função hipotálamo-hipofisária (megateste) pré-operatórios. Todos os tumores tiveram diagnóstico histológico e em 17 foi realizado também estudo imuno-histoquímico para os hormônios adeno-hipofisários. A análise estatística foi feita por meio dos testes t de Student, qui-quadrado, exato de Fisher e de Mc Neman. O nível de significância adotado foi $5 \%(p<0,05)$. Resultados: 0 diâmetro tumoral variou de 1,1 a 4,7 cm (média=2,99 cm $\pm 1,04)$. No pré-operatório, $5(21,7 \%)$ pacientes não apresentaram déficit hormonal laboratorial, $9(39,1 \%)$ desenvolveram hiperprolactinemia, $13(56,5 \%)$ níveis normais de prolactina (PRL) e 1 (4,3\%) subnormal; 18 $(78,3 \%)$ pacientes desenvolveram hipopituitarismo (4 pan-hipopituitarismo). Dezenove pacientes $(82,6 \%)$ foram submetidos a via de acesso transesfenoidal, 3 (13\%) a craniotomia e $1(4,4 \%)$ ao acesso combinado. Somente 6 pacientes tiveram ressecção tumoral total. Dos 17 submetidos a estudo imuno-histoquímicos, 5 tumores eram imunonegativos, 1 misto, $1 \mathrm{LH}+, 1 \mathrm{FSH}+, 1$ sub-unidade alfa e 8 apresentaram imunorreatividade focal ou em células isoladas para um dos hormônios adeno-hipofisários ou sub-unidades; dos outros seis tumores, 5 eram cromófobos e 1 cromófobo/acidófilo. Não houve correlação estatisticamente significativa entre o diâmetro tumoral e os níveis de PRL pré-operatória $(p=0,82)$, nem entre tamanho tumoral e o estado hormonal pós-operatório, exceto nos eixos GH e gonadotrófico. Foi observada significância estatística entre o tamanho tumoral e o estado hormonal pré-operatório (exceto no eixo gonadal); entre níveis normais de PRL, associados a discreta ou a nenhuma disfunção hipofisária pré-operatória, e recuperação da função hipofisária pós-operatória. Conclusão: A hiperprolactinemia e tamanho tumoral pré-operatórios isolados não foram preditivos para a recuperação da função hipofisária pós-operatória.
\end{abstract}

PALAVRAS-CHAVE: glândula pituitária, adenomas, hiperprolactinemia, hipopituitarismo.

Influence of hyperprolactinemia and tumoral size in the postoperative pituitary function in clinically nonfuncioning pituitary macroadenomas

ABSTRACT- Objective: To study the influence of hyperprolactinemia and tumoral size in the pituitary function in clinically nonfuncioning pituitary macroadenomas. Methods: Twenty three patients with clinically nonfuncioning pituitary macroadenomas were evaluated by image studies (computed tomography or magnetic resonance) and basal hormonal level; 16 had preoperative hypothalamus-hypophysial function tests (megatests). All tumors had histological diagnosis and in seventeen immunohistochemical study for adenohypophysial hormones was also performed. Student's t test, chi square test, exact test of Fisher and Mc Neman test were used for the statistics analysis. The level of significance adopted was $5 \%(p<0.05)$. Results: Tumoral diameter varied of 1.1 to $4.7 \mathrm{~cm}$ (average $=2.99 \mathrm{~cm} \pm 1.04$ ). In the preoperative, $5(21.7 \%)$ patients did not show laboratorial hormonal deficit, $9(39.1 \%)$ developed hyperprolactinemia, $13(56,5 \%)$ normal levels of prolactin (PRL) and 1 (4.3\%) subnormal; 18 (78.3\%) patients developed hypopituitarism (4 pan-hypopituitarism). Nineteen patients (82.6\%) underwent transsfenoidal approach, 3 (13\%) craniotomy and 1 (4.4\%) combined access. Only 6 patients had total tumoral resection. Of the 17 immunohistochemical studies, 5 tumours were

\footnotetext{
${ }^{1}$ Médica do Serviço de Neurocirurgia do Hospital Universitário Clementino Fraga Filho (HUCFF), Universidade Federal do Rio de Janeiro (UFRJ) Rio de Janeiro RJ, Brasil; ${ }^{2}$ Professora Titular e Chefe de Departamento de Patologia, Faculdade de Medicina (FM/UFRJ); ${ }^{3} \mathrm{Chefe}$ Médico do Laboratório de Hormônios HUCFF; ${ }^{4}$ Professor Adjunto e Chefe do Departamento de Radiologia da Universidade Federal Fluminense (UFF), Niterói RJ, Brasil; ${ }^{5}$ Professora Adjunta da Disciplina de Endocrinologia, FM/UFRJ.
}

Recebido 28 Novembro 2001, recebido na forma final 2 Março 2002. Aceito 13 Março 2002.

Dra. Ana Luiza Vidal Fonseca - Serviço de Neurocirurgia HUCFF/UFR - Av. Trompowsky s/n, Ilha do Fundão - 21941-590 Rio de Janeiro RJ - Brasil. 
immunonegatives, 1 compound, $1 \mathrm{LH}+, 1 \mathrm{FSH}+, 1$ alpha sub-unit and 8 focal or isolated immunorreactivity for one of the pituitary hormones or sub-units; of the other six tumours, 5 were chromophobe and 1 chromophobe/acidophile. No significanct statistic difference was noted between tumoral size and preoperative PRL levels $(p=0.82)$, nor between tumoral size and postoperative hormonal state, except in the GH and gonadal axis. Significant statistic was noted: between tumoral size and preoperative hormonal state (except in the gonadal axis); between normal PRL levels, associated to none or little preoperative hypophysial disfunction, and recovery of postoperative pituitary function. Conclusion: Isolated preoperative hyperprolactinemia and tumoral size have not been predictible for the recovery of postoperative pituitary function.

KEY WORDS: pituitary gland, adenomas, hyperprolactinemia, hypopituitarism.

Várias doenças afetam a região selar e áreas adjacentes, correspondendo o adenoma hipofisário a $90 \%$ delas. Esses tumores podem causar síndrome de hipersecreção (70\% dos adenomas hipofisários) e/ou hiposecreção hormonal (hipopituitarismo), distúrbios neurológicos e/ou hipertensão intracraniana ${ }^{1}$. A disfunção endócrina, se não tratada, pode evoluir para complicações clínicas diversas, incluindo abolição da elevação normal e fisiológica de glicocorticóides em resposta ao estresse, aterosclerose acelerada, hipertensão arterial, miopatia cardíaca, osteoporose e diabetes mellitus. Isso aumenta a morbidade e mortalidade desses pacientes ${ }^{2,3}$. $O$ tratamento cirúrgico nem sempre resulta em regressão clínica e/ou laboratorial dos distúrbios hormonais pré-operatórios.

Em adenomas hipofisários clinicamente não-funcionantes, os fatores preditivos para recuperação do hipopituitarismo, segundo $\operatorname{Arafah}^{4}$, seriam o tamanho tumoral e a hiperprolactinemia, o que não tem sido encontrado por outros autores ${ }^{5,6}$. A partir desta discordância nos propusemos a estudar este assunto.

\section{MÉTODO}

Foram estudados pacientes com tumores hipofisários clinicamente não funcionantes, acompanhados nos Ambulatórios de Endocrinologia e/ou de Neurocirurgia do Hospital Universitário Clementino Fraga Filho (HUCFF) da Universidade Federal do Rio de Janeiro (UFRJ), de janeiro de 1980 a abril de 2001 . Foram operados 44 pacientes com adenomas clinicamente não-funcionantes (36,4\% dos tumores hipofisários) avaliados nesse período. Após revisão dos prontuários, 21 pacientes foram excluídos: 2 com microadenomas, 9 sem dosagem hormonal pré- e/ou pósoperatória e pré-radioterapia, 7 sem descrição do diâmetro tumoral, 4 sem comprovação histológica de adenomas hipofisários e 1 por ter evoluído para apoplexia.

A avaliação hormonal foi feita através da dosagem basal dos hormônios hipofisários (LH, FSH, PRL, GH, TSH), cortisol, hormônios tireoidianos e esteróides gonadais. $\mathrm{O}$ teste de função integrada hipotálamo-hipofisária (megateste) foi obtido por:

1. estímulo de LH e FSH com $100 \mathrm{mg}$ endovenosa de LHRH (hormônio liberador de gonadotropinas), amostras de sangue colhidas 30, 60 e 90 minutos após a injeção (resposta normal se houver, no mínimo, um pico do dobro do basal para o $\mathrm{LH}$ aos 30 minutos, e a resposta do FSH variando de $50-200 \%$ do valor basal);

2. estímulo de TSH e PRL com $200 \mu \mathrm{g}$ endovenosa de TRH (hormônio liberador de TSH), amostras de sangue coIhidas 30, 60 e 90 minutos após a injeção (resposta normal - aumento de 2-3 vezes do valor basal da PRL e pico de TSH, 20-30 minutos após a injeção, seguido de queda gradativa);

3. estímulo do cortisol e GH com hipoglicemia induzida por 0,05 a 0,1 Ul/kg de insulina regular endovenosas com amostras de sangue colhidas 30, 60 e 90 minutos após a injeção; o teste é considerado válido se há hipoglicemia clínica ou laboratorial (glicemia $\leq 40 \mathrm{mg} / \mathrm{dl}$ ); resposta normal do GH- pico acima de $5 \mathrm{ng} / \mathrm{ml}$ em qualquer amostra; resposta normal do cortisol - aumento de $7 \mu \mathrm{g} / \mathrm{dl}$ do valor basal ou $>17 \mu \mathrm{g} / \mathrm{dl}$ em qualquer tempo)

Todos tinham dosagens dos hormônios hipofisários ( $\mathrm{LH}$, FSH, PRL, GH, TSH), cortisol, hormônios gonadais. Durante o período no qual os 23 pacientes foram acompanhados, houve variação dos valores hormonais normais, devido a diferença de material de análise e métodos laboratoriais empregados (radioimunoensaio e imunoensaio por quimioluminescência). Por esse motivo, a análise estatística dos valores dos hormônios não foi feita quantitativamente e sim qualitativamente (aumentado, normal ou diminuído).

Todos os tumores tiveram diagnóstico histológico e 17 deles estudo imuno-histoquímico para hormônios adeno-hipofisários e sub-unidade alfa. Todos os pacientes estudados tinham TC pré-operatória e 13 deles fizeram RM. Dos 23 pacientes, foram avaliadas 20 imagens; os 3 restantes, tinham descrição dos laudos em seus prontuários que incluiam o maior diâmetro e o tipo de extensão tumoral. Um paciente não recebeu contraste por história de reação alérgica grave.

Esse estudo foi submetido ao Comitê de Ética da Comissão de Investigação Científica (CIC) do HUCFF (UFRJ) e aceito. Todos os pacientes assinaram termo de consentimento para que os dados pudessem ser divulgados. A análise estatística foi feita através dos testes t de Student, Quiquadrado, exato de Fisher e de Mc Neman, sob orientação da CIC. O nível de significância adotado foi $5 \%(p<0,05)$.

\section{RESULTADOS}

A maioria dos pacientes foi operada entre 19922001, exceto um caso (1986). Dos 23 pacientes com 
macroadenomas clinicamente não-funcionantes analisados, 15 (65\%) eram homens e 8 (35\%) mulheres. A mediana da idade foi 47,7 anos (18-71 anos); 11 tinham 50 anos ou mais. O tempo de início dos sintomas variou de 6 meses a 8 anos (média $=2,5$ anos), sendo em um caso achado radiológico. As principais queixas foram: disfunção visual $(78,2 \%)$, cefaléia $(39,1 \%)$, impotência $(26,1 \%)$, disfunção menstrual $(21,7 \%)$, galactorréia $(17,4 \%)$, cansaço/adinamia $(17,4 \%)$ e diplopia (13\%). Dos dezoito casos de disfunção visual, 2 apresentavam amaurose, 2 diminuição da acuidade visual e o restante hemianopsia bitemporal. Uma paciente tinha seqüela de encefalomielite pós-rubéola, mas nenhum outro paciente tinha qualquer doença ou fazia uso de medicamentos em dosagens que pudessem alterar os valores hormonais.

No pré-operatório, apenas 5 pacientes $(21,7 \%)$ não apresentaram qualquer deficiência hormonal e 4 $(17,4 \%)$ pan-hipopituitarismo. Nos 18 pacientes (78,3\%) com hipopituitarismo, a deficiência hormonal mais freqüente foi a do GH, seguida por TSH e FSH/ LH. (Tabela 1). A concentração de PRL, em números absolutos, variou de 4,9 a $76,0 \mathrm{ng} / \mathrm{ml}$; a representação qualitativa pré-operatória, de acordo com o diâmetro tumoral, encontra-se na Figura 1. Nenhum apresentou diabetes insipidus pré-operatório.

Das 20 imagens avaliadas, 11 tumores eram espontaneamente isodensos/isointensos (55\%) e 9 hiperdensos/hiperintensos (45\%), em relação ao parênquima cerebral, respectivamente à TC e/ou RM. Impregnaram-se moderadamente pelo contraste 11 lesões $(57,9 \%)$ e, intensamente, $8(42,1 \%)$. Expansão supra-selar ocorreu em 17/20 (85\%), sendo 4 associadas com expansão infra-selar e 1 para-selar. Existia compressão do quiasma óptico em 16 (80\%) e do III ventrículo em 8 (40\%). A haste foi identificada em apenas 3 casos (15\%), sendo desviada em um. Quanto à forma, 14 lesões (70\%) eram simétricas e 6 assimétricas (30\%). Outras características encontradas: assoalho adelgaçado em 9 casos (45\%), erodido em 7 (35\%), necrose ou pequeno cisto intratumoral em 7 (35\%) e microcalcificações em 2 (10\%), estas últimas demonstradas à TC.

Quanto ao tipo de cirurgia, 19 pacientes $(82,6 \%)$ foram submetidos a via de acesso transesfenoidal, 3 $(13 \%)$ a craniotomia e $1(4,4 \%)$ a acesso combinado em dois tempos (transesfenoidal e craniotomia). Complicações pós-operatórias: sinusite (5 casos), perfuração de septo (2), fístula liquórica (2), hiponatremia (1), sinéquia nasal (1) e infecção da craniotomia (1).

Dos 17 diagnósticos imuno-histológicos, 5 (29,4\%) eram tumores imunonegativos, $1(5,9 \%)$ misto (FSH/LH/

Tabela 1. Perfil hormonal qualitativo pré- e pós-operatórios.

\begin{tabular}{cccc}
\hline Hormônio & Normal & Diminuído & Elevado \\
\hline Pré-Operatório & & & \\
PRL & $13(56,5)$ & $1(4,3)$ & $9(39,1)$ \\
GH & $11(47,8)$ & $12(52,2)$ & 0 \\
TSH/T4L & $12(52,2)$ & $11(47,8)$ & 0 \\
FSH/LH & $13(56,5)$ & $10(43,5)$ & 0 \\
CORTISOL & $15(65,2)$ & $8(34,8)$ & 0 \\
Pós-Operatório & & & $8(34,8)$ \\
PRL & $12(52,2)$ & $3(13,0)$ & 0 \\
GH & $11(47,8)$ & $12(52,2)$ & 0 \\
FSH/LH & $13(56,5)$ & $10(43,5)$ & 0 \\
TSH/T3/T4L & $15(65,2)$ & $8(34,8)$ & 0 \\
\hline CORTISOL & $15(65,2)$ & $8(34,8)$ & \\
\hline
\end{tabular}

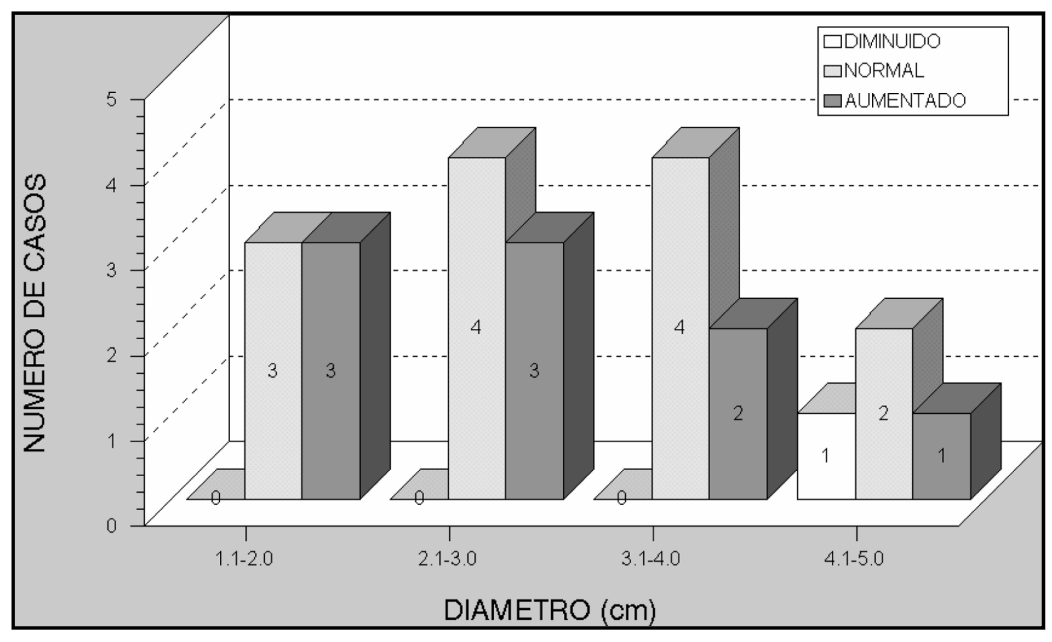

Fig 1. Estado da prolactina pré-operatória de acordo com o diâmetro tumoral. 
Tabela 2. Significância estatística entre diâmetro tumoral préoperatório e condições hormonais pré e pós-operatórias.

\begin{tabular}{lcc}
\hline & Pré-operatório & Pós-operatório \\
GH & $p=0,001$ & $p=0,03$ \\
Cortisol & $p=0,005$ & $p=0,18$ \\
TSH & $p=0,009$ & $p=0,06 *$ \\
FSH/LH & $p=0,10$ & $p=0,02$ \\
\hline
\end{tabular}

Em itálico =correlação estatística; * tendência a significância estatística

ACTH isolada para PRL/TSH), 1 (5,9\%) LH+ (focal para FSH e TSH), 1 (5,9\%) FSH+, 1 sub-unidade alfa $(5,9 \%)$ e 8 (47\%) apresentaram imunorreatividade focal ou em células isoladas para um dos hormônios adeno-hipofisários ou sub-unidades. Dos seis tumores restantes, 5 eram cromófobos e 1 cromófobo/acidófilo.

O tempo de acompanhamento ambulatorial, da data da cirurgia até a última consulta, variou entre 4 meses e 6 anos (média de 2,7 anos). No pós-operatório, $17(73,9 \%)$ pacientes tinham lesão residual ao exame de imagem, realizado em 3 meses a 1 ano de pósoperatório. Destes, 16 (69,5\%) apresentavam hipopituitarismo (4 pan-hipopituitarismo), 14 dos quais necessitaram de reposição de um ou mais hormônios: corticosteróide (11), hormônio tireoidiano (7), esteróides gonadais (6). Diabetes insipidus transitório pósoperatório ocorreu em 15 pacientes $(65,2 \%)$. Sete pacientes foram reoperados e um paciente faleceu por progressão da doença. Radioterapia foi indicada em 12 casos $(52,2 \%)$ por crescimento da lesão residual.

O diâmetro tumoral pré-operatório variou de 1,1 a $4,7 \mathrm{~cm}$ (média $=2,99 \mathrm{~cm} \pm 1,04$ ). Os pacientes foram subdivididos em 4 grupos: 6 com tumores entre 1,1 e $2 \mathrm{~cm}, 7$ entre 2,1 e $3 \mathrm{~cm}, 6$ entre 3,1 e $4 \mathrm{~cm}$ e $4 \mathrm{com}$ tumores maiores que $4 \mathrm{~cm}$. Os pacientes de sexo masculino apresentaram os maiores tumores; dos 10 pacientes que tinham tumores com mais de $3 \mathrm{~cm}$ de diâmetro, apenas um era do sexo feminino. O diâmetro tumoral não se correlacionou estatisticamente com os níveis de PRL $(p=0,82)$.

$A$ variação do número de eixos acometidos de acordo com o aumento do diâmetro tumoral, não foi uniforme; pan-hipopituitarismo só ocorreu em um caso dos tumores com diâmetro maior que 4 $\mathrm{cm}$. O diâmetro tumoral também foi comparado com o estado pré- e pós-operatório de cada hormônio (normal ou deficiente). Houve correlação estatisticamente significativa entre diâmetro tumoral e o estado hormonal pré-operatório (exceto no eixo gonadal), mas não com estado hormonal pós-operatório (exceto os eixos GH e gonadal) (Tabela 2).

A PRL pré-operatória, dividida em dois grupos normal e aumentada, foi comparada com o estado de cada hormônio pós-operatório (normal e diminuído). Não houve significância estatística entre a PRL pré-operatória e estado hormonal pós-operatório (Tabela 3). Em seguida, o grupo de PRL normal no pré-operatório, foi dividido em dois sub-grupos, na tentativa de estabelecer se o valor era realmente normal ou se o paciente estava apresentando queda da hiperprolactinemia por lesão dos lactotrofos; N1 - associada a ausência de disfunção pré-operatória dos outros eixos ou a deficiência de apenas um eixo; N2 associada a disfunção pré-operatória de dois ou mais eixos hormonais. Esses três sub-grupos de PRL pré-operatórias (N1, N2 e aumentada) foram comparados com o estado hormonal pós-operatório e só então mostrou correlação significativa entre eles (exceto no eixo do cortisol) (Tabela 3).

Tabela 3. Nível de significância entre a PRL pré-operatória e a normalidade hormonal pós-operatória.

\begin{tabular}{lccccc}
\hline PRL pré operatória & Total & $\begin{array}{c}\text { GH-PO } \\
\text { normal }\end{array}$ & $\begin{array}{c}\text { Cortisol-PO } \\
\text { normal }\end{array}$ & $\begin{array}{c}\text { LH/FSH-PO } \\
\text { normal }\end{array}$ & $\begin{array}{c}\text { TSH/T4L/T3-PO } \\
\text { normal }\end{array}$ \\
\hline Diminuída & 1 & - & - & - & - \\
Aumentada & 9 & $6 / 9(66,7 \%)$ & $7 / 9(77,78 \%)$ & $7 / 9(77,78 \%)$ & $8 / 9(88,89 \%)$ \\
Normal (N1 + N2) & 13 & $5 / 13(38,46 \%)$ & $8 / 13(61,54 \%)$ & $6 / 13(46,15 \%)$ & $7 / 13(53,85 \%)$ \\
Nível de significância & & 0,19 & 0,37 & 0,14 & 0,10 \\
N1 & 6 & $4 / 6(66,67 \%)$ & $4 / 6(66,67 \%)$ & $5 / 6(83,33)$ & $5 / 6(83,33)$ \\
Aumentada & 9 & $6 / 9(66,7 \%)$ & $7 / 9(77,78 \%)$ & $7 / 9(77,78 \%)$ & $8 / 9(88,89 \%)$ \\
N2 & 8 & $1 / 8(12,5 \%)$ & $4 / 8(50 \%)$ & $1 / 8(12,5 \%)$ & $2 / 8(25 \%)$ \\
Nível de significância & & 0,04 & 0,48 & 0,009 & 0,01 \\
\hline
\end{tabular}

Em itálico, correlação estatística, PO, pós-operatório; N1, nível normal sem comprometimento das células hipofisárias; N2, nível normal com comprometimento das células hipofisárias. 


\section{DISCUSSÃO}

Hiperprolactinemia e hipopituitarismo causam morbidade variada e, por isso, devem ser identificados e tratados precocemente ${ }^{3,7,8}$. O prognóstico de adenomas hipofisários, quanto à função hipofisária pós-operatória, tem sido descrito de forma contraditória. Arafah et al. ${ }^{9}$ descreveram que a persistência do hipopituitarismo pós-operatório seria resultante de isquemia ou necrose hipofisária, pelo tempo de compressão hipofisária.

Analisamos 23 pacientes com macroadenomas clinicamente não-funcionantes. A heterogeneidade de informações das imagens não comprometeram o objetivo desse estudo, de correlacionar tamanho tumoral (maior diâmetro tumoral) e níveis de prolactina. Nossos resultados foram similares aos descritos na literatura, exceto na taxa de lesão residual e de diabetes insípidus (Tabela 4). Essas diferenças existiram, provavelmente, porque nosso estudo só analisou macroadenomas, sendo a média do diâmetro tumoral foi de $2,99 \mathrm{~cm}( \pm 1,04), 4 / 23(17,4 \%)$ dos quais eram gigantes. Na literatura, a frequência de adenomas gigantes ( $>4 \mathrm{~cm}$ de diâmetro) é $6 \%$.

Sabe-se que a experiência do cirurgião interfere nos resultados relativos à função hipofisária pósoperatória, pois quanto mais experiente, maior a ca-

Tabela 4. Comparação entre nosso estudo e a literatura.

\begin{tabular}{lcc}
\hline Incidência dos TNF & Nosso estudo & Literatura \\
\hline Tumores hipofisários & $36,4 \%$ & $10-40 \%$ \\
Sexo masculino & 2 & $1-3$ \\
$\begin{array}{l}\text { Idade mediana ao } \\
\text { diagnóstico }\end{array}$ & 47,7 anos & $>40$ anos \\
Disfunção visual & $78,2 \%$ & $48-72,4 \%$ \\
Hiperprolactinemia & $39,1 \%$ & $33-47 \%$ \\
Hipopituitarismo & $78,3 \%$ & $44-77 \%$ \\
$\begin{array}{l}\text { Imunoreatividade focal ou } \\
\text { isolada para uma sub- } \\
\text { unidade dos hormônios } \\
\text { glicoprotéicos }\end{array}$ & & \\
Lesão residual & $64,3 \%$ & $70-100 \%$ \\
$\begin{array}{l}\text { Taxa de recorrência } \\
\text { anterior a RT }\end{array}$ & $73,9 \%$ & $10-42 \%$ \\
$\begin{array}{l}\text { Hipopituitarismo } \\
\text { pós-operatório }\end{array}$ & & $8-71 \%$ \\
Diabetes insipidus & $52,2 \%$ & $23-88 \%$ \\
\hline *adenomas hipofisaros & $69,5 \%$ & \\
\hline
\end{tabular}

*adenomas hipofisários em geral pacidade de escolher a via de abordagem cirúrgica mais apropriada, identificar o tecido hipofisário normal, não tracionar excessivamente a haste, avaliar o grau de ressecção e identificar o tecido adenomatoso extra-selar ${ }^{1,7}$. O estudo atual foi feito em ambiente universitário e isso pode ter contribuído para alta taxa de lesão residual e diabetes insípidus. Paradoxalmente, só tivemos um óbito por evolução da doença, não tivemos nenhuma complicação grave e todos os casos de diabetes insipidus foram transitórios. Nessa casuística, o diâmetro tumoral não se correlacionou com os níveis de PRL. O diâmetro tumoral e os níveis de PRL não se correlacionaram com hipopituitarismo pós-operatório. A significância estatística ocorreu, para a recuperação da função pós-operatória, quando os níveis de prolactina foram associados a função normal ou a disfunção de apenas um eixo hormonal.

Não é difícil imaginar que, quanto maior o volume tumoral maior seria a probabilidade de compressão da haste hipofisária e da hipófise, resultando em disfunção hormonal. Arafah descreveu, em vários trabalhos desde 1986, que os altos níveis de prolactina pré-operatórios e tumores menores ou iguais a $25 \mathrm{~mm}$ de diâmetro são preditivos de bom prognóstico para recuperação da função hipofisária ${ }^{4,9-13}$. Os mesmos achados não foram encontrados por outros autores, incluindo o estudo presente ${ }^{5,6,14}$. Klijn et al. ${ }^{15}$ apesar de terem encontrado correlação entre os níveis de prolactina e o tamanho tumoral, não separaram prolactinomas dos tumores não funcionantes (TNF). Smith et al. ${ }^{6}$, em 44 casos de pseudoprolactinomas por RM, não encontraram correlação significante entre níveis de PRL e o desvio angular e/ ou compressão da haste; teorizaram que o principal fator para a disfunção hormonal seria o aumento da pressão intra-selar. Greenman et al. ${ }^{5}$ compararam 26 macroadenomas não-funcionantes com 15 macroadenomas secretores de $\mathrm{GH}$; em sua série ocorreu prevalência maior e mais grave do hipopituitarismo em TNF do que em macroadenomas somatotrófico, porém não houve diferença estatisticamente significativa dos níveis de PRL entre os dois grupos, nem correlação entre tamanho e função hipofisária.

No efeito de secção da haste, os níveis de prolactina aumentam e dos outros hormônios diminuem, progressivamente, com o tamanho tumoral ${ }^{1,3}$. Quando os lactotrofos normais são lesados pela compressão tumoral, os níveis de prolactina declinam. Portanto, os níveis normais ( $\mathrm{N}$ ) e aumentados de prolactina (hiperprolactinemia $=\mathrm{H}$ ) refletem dois mo- 

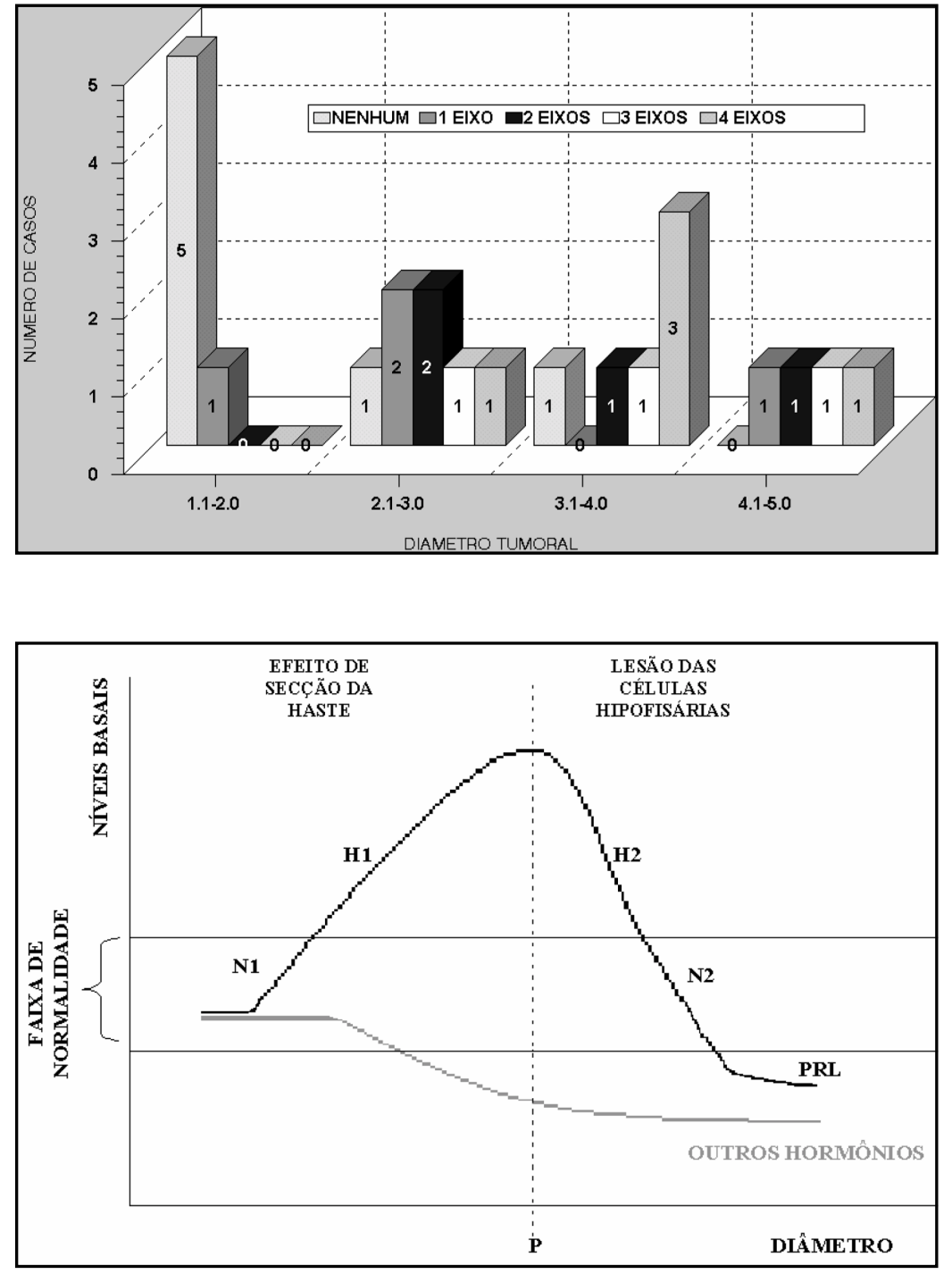

Fig 2. Número de eixos hipofisários acometidos no pré-operatório de acordo com o diâmetro tumoral.
Fig 3. Variação teórica dos níveis hormonais com o tamanho tumoral. $N_{1}$, normal real- sem compressão da haste ou início da compressão da haste; $\mathrm{H1}$,compressão da haste; $\mathrm{H}_{2^{\prime}}$ hiperprolactinemia com comprometimento dos lactotrofos; $N_{2}$, normal aparente- comprometimento dos lactotrofos, resultando em diminuição dos níveis de PRL. mentos diferentes (Fig 1). Essa pode ser uma explicação para diferentes resultados quanto à correlação entre volume tumoral e disfunção hormonal, encontrados na literatura. No presente estudo, apesar dos sub-grupos terem número pequeno de casos, pode-se observar nas Figuras 1 e 2 a falta de uniformidade de resultados hormonais entre eles.

Apesar da importância dada ao efeito de secção da haste, ela, por si só, não pode explicar: 1. ausência de correlação entre hiperprolactinemia e tamanho tumoral em pseudoprolactinomas ${ }^{6} ; 2$. níveis variáveis de hiperprolactinemia, em secção terapêutica ou experimental da haste feitas no passado ${ }^{16,17}$; 3. a falta de concomitância entre hiperprolactinemia e hipopituitarismo, em massas expansivas selares, diferente do que ocorre quando a haste é seccionada experimentalmente ${ }^{6} ; 4$. os tumores de tamanho similar com diferentes expressões, clínica e laboratorial, encontrados no dia a dia; 5 . sela vazia associa- da com hiperprolactinemia e haste visivelmente estirada, frequentemente sem hipopituitarismo ${ }^{6} ; 6$. a presença de adenoma gigante com discreto distúrbio hormonal ${ }^{14} ; 7$. a presença de macroincidentaloma ${ }^{1,29}$, assim como a apoplexia hipofisária em macroadenomas sem história prévia de endocrinopatia $^{1} ; 8$. a presença de hiperprolactinemia em pacientes com microadenomas TNF (32\% dos TNF) ${ }^{18} ; 9$. a prevalência maior de hipopituitarismo grave, pré e pós-operatórios, em macroadenomas-TNF do que macroadenomas secretores de $\mathrm{GH}^{5}$

Em estudos experimentais e clínicos, os motivos supostos para explicar essa variação dos níveis de prolactina entre TNF e lesões expansivas não hipofisárias foram: 1. rotas alternativas da liberação de dopamina para a adeno-hipófise, além do sistema portal curto através da neuro-hipófise; 2 . secção incompleta ou somente distorção parcial da haste pelo tumor, preservaria o sistema de liberação de dopa- 
mina da neuro-hipófise; 3 . liberação de prolactina dos lactotrofos lesados; 4 . aumento da pressão intraselar (PIS) 6,19,20.

Em 1987, Lees et al. ${ }^{20}$ e, em 2000, Arafah et al. ${ }^{9}$ demostraram que a pressão intra-selar média foi mais alta em pacientes com hiperprolactinemia comparados com aqueles de prolactina normal. Não se sabe qual o valor normal da pressão intra-selar, mas é provavel que não exceda a pressão intracraniana ${ }^{6,9}$. A pressão de perfusão dos sistemas portais deve ser consideravelmente mais baixa do que os tecidos que recebem suprimento arterial direto e o aumento na pressão intra-selar pode alterar a comunicação entre o hipotálamo e a adeno-hipófise por mudanças no fluxo sanguíneo e no transporte axonal ${ }^{4,20}$. Aumento da pressão intra-selar causando disfunção hormonal da adeno-hipófise pode ser influenciada por vários fatores: 1. manobra de Valsalva, pré e/ou per-operatórias causaria aumento agudo da pressão intra-selar (demonstrada por Lees et al. $)^{20}$ e poderia resultar em grau variável de necrose da adenohipófise; b) elevação transitória da pressão venosa, demonstrada experimentalmente, por Doppler, em primatas (citado por Arafah et al.) ${ }^{9}$; c) velocidade de expansão de lesões intra-selares, permitindo maior ou menor adaptação às variações de pressão (exemplo, tumor $x$ apoplexia) ${ }^{1,3}$; d) variação anatômica da região selar pode criar maior ou menor resistência a expansão tumoral intra-selar, contribuindo ou não para aumento precoce da pressão intra-selar ${ }^{21}$; e) absorção óssea, em pacientes de idade avançada, pode facilitar a erosão e expansão do tumor, antes de causar pressão hipofisária grave ${ }^{1}$; f) densidade da lesão ${ }^{6}$; g) associação de adenomas com outras lesões ${ }^{22}$.

Outros fatores podem contribuir para a hiperprolactinemia e/ou hipopituitarismo, gerando resultados conflitantes na literatura. Podem influenciar o eixo hipotálamo-hipofisário diversos fatores exógenos e endógenos (idade, sexo, condições fisiológicas, doenças dos órgãos alvos, medicamentos, etc) ${ }^{1,23}$, TNF com imunorreatividade isolada para PRL ${ }^{11,24}$, secreção ectópica de prolactina ${ }^{23,25}$, nem toda a prolactina circulante é biologicamente ativa ${ }^{19,26,27}$; casos raros de adenomas múltiplos ${ }^{28}$, presença de hipófise faríngea (não se sabe qual o grau de funcionabilidade dessas células) ${ }^{1}$.

Conclui-se que o tamanho tumoral e os níveis de PRL pré-operatórios em TNF não se correlacionam com a função hipofisária no pós operatório e, portanto, não podem ser considerados fatores preditivos para a recuperação da função.

\section{REFERÊNCIAS}

1. Krisht AF, Tindall GT. Pituitary disorders: comprehensive management. Baltimore: Lippincott Williams \& Wilkins, 1999:99-118.

2. Greenspan FS, Strewler GJ. Basic and clinical endocrinology. In Hypothalamus and pituitary. Stamford, UK: Appleton \& Lange, 1997:95-156.

3. Tindall GT, Cooper PR, Marrow DL. The practice of neurosurgery. Baltimore: Lippincott Williams \& Wilkins, 1996:1021-1158.

4. Arafah BM. Reversible hypopituitarism in patients with large nonfunctioning pituitary adenomas. J Clin Endocrinol Metab 1986;62:1173-1179.

5. Greenman Y, Tordjman K, et al. Relative sparing of anterior pituitary function in patients with growth hormone-secreting macroadenomas: comparison with nonfunctioning macroadenomas. J Clin Endocrinol Metab 1995;80:1577-1583.

6. Smith MV, Laws ER Jr. Magnetic resonance imaging measurements of pituitary stalk compression and deviation in patients with nonprolactinsecreting intrasellar and parasellar tumors: lack of correlation with serum prolactin levels. Neurosurgery 1994;34:834-839.

7. Vance ML. Hypopituitarism. N Engl J Med 1994;330:1651-1662.

8. Tomllinson JW, Holden N, Hills RK, et al. Association between premature mortality and hypopituitarism. Lancet 2001;357:25-31.

9. Arafah BM, Prunty D, Ybarra J, Hlavin ML, Selman WR. The dominant role of increased intrasellar pressure in the pathogenesis of hypopituitarism, hyperprolactinemia, and headaches in patients with pituitary adenomas. J Clin Endocrinol Metab 2000;85:1789-1793.

10. Arafah BM, Harrington JF, Madhoun ZT, et al. Improvement of pituitary function after surgical decompression for pituitary tumor apoplexy. J Clin Endocrinol Metab 1990;71:323-328.

11. Arafah BM, Kailani SH, Nekl KE, et al. Immediate recovery of pituitary funtion following transsphenoidal ressection of pituitary macroadenomas. J Clin Endocrinol Metab 1994;79:348-354.

12. Arafah BM, Nekl KE, Gold RS, et al. Dynamics of prolactin secretion in patients with hypopituitarism and pituitary macroadenomas. J Clin Endocrinol Metab 1995;80:3507-3512.

13. Arafah BM, Selman WR. Perioperative endocrine management of pituitary adenomas. Tech Neurosurg 2000;6:248- 257.

14. Goel A, Nadkarni T. Surgical management of giant pituitary tumours: a review of 30 cases. Acta Neurochir (Wien) 1996;138:1042-1049.

15. Klijn JGM, Lamberts SWJ, DeJong FH, et al. The importance of pituitary tumor size in patients with hyperprolactinemia in relation to hormonal variables and extrasellar extension of tumor. Clin Endocrinol 1980;12:341-355.

16. Diefenbach WMP, Carmel PW, Frantz AG, et al. Supression of prolactin secretion by L-dopa in the stalk-sectioned rhesus monkey. J Clin Endocrinol Metab 1976;43:638-642.

17. Field RA, Hall WA, Contreras JS, et al. Hypophyseal stalk section in the treatment of advancing diabetic retinopathy: report of three cases. N Engl J Med 1961;264:689-698.

18. Drange MR, Fram NR, Bonert HV, et al. Pituitary tumor registry: a novel clinical resource. J Clin Endocrinol Metab 2000;85:168-174.

19. Albuquerque FC, Hinton DR, Weiss MH. Excessively high prolactin level in a patient with a nonprolactin-secreting adenoma. J Neurosurg 1998;89:1043-1046.

20. Lees PD, Pickard JD. Hyperprolactinemia, intrasellar pituitary tissue pressure, and the pituitary stalk compression syndrome. J Neurosurg 1987;67:192-196.

21. Henriques JGB, Pianetti G. Anatomia microcirúrgica da região selar em cadáveres a fresco utilizando técnica fotográfica com fluoresceína. Arq Neuropsiquiatr 2000;58:485-493.

22. Weir B. Pituitary tumors and aneurysms: case report and review of the literature. Neurosurgery 1992;30:585-590.

23. Molitch ME. Disorders of prolactin secretion. Endocrinol Metab Clin N Am 2001;30:585-610.

24. Landeiro JA, Flores MS, Braga FM, et al Macroadenomas não-funcionantes da hipófise, estudo imuno-histoquímico. Arq Br Neurocir 1996;15:181-186.

25. Herzog AG. Migraine with ectopic hyperprolactinemia from uterine fibroids. Neurology 2000;55:148-149.

26. Cavaco B, Leite V, Santos MA, et al. Some forms of big big prolactin behave as a complex of monomeric prolactin with an immunoglobulin $\mathrm{G}$ in patients with macroprolactinemia or prolactinoma. J Clin Endocrinol Metab 1995;80:2342-2346.

27. Tritos NA, Guay AT, Malarkey WB. Asymptomatic 'big' hyperprolactinemia in two men with pituitary adenomas. Eur J Endocrinol 1998;138:82-85.

28. Tosaka M, Kohga H, Kobayashi S, et al. Double pituitary adenomas detected on preoperative magnetic resonance images. J Neurosurg 2000;92:361.

29. Aron DC, Howlett TA. Endocrine Incidentalomas: pituitary incidentalomas. Endocrinol Metab Clin 2000;29:205-221. 